\title{
Inflammation In HSC Emergence
}

\author{
Roshana Thambyrajah* \\ Stem Cell and Cancer Group, IMIM, PRBB, Barcelona, Spain \\ *Corresponding author: Roshana Thambyrajah, Stem Cell and Cancer Group, IMIM, PRBB, Barcelona, Spain
}

\begin{tabular}{|c|c|}
\hline ARTICLE INFO & ABSTRACT \\
\hline Received: 幽 August 28, 2021 & $\begin{array}{l}\text { Citation: Roshana Thambyrajah. Inflammation In HSC Emergence. Biomed J Sci \& Tech } \\
\text { Res 39(2)-2021. BJSTR. MS.ID.006276. }\end{array}$ \\
\hline
\end{tabular}

\section{Opinion}

In our classical view, inflammation is triggered by environmental changes; may it be pathogens or tissue damage. The stimuli activate a complex transcriptional program that is both cell type and stimulus specific. At the cellular level, a complex gene expression programs that include hundreds of genes is activated within minutes after the initial activation through a stimulus [1]. But this response can vary not only between cell types [2], but even within a cell population depending on their niche environment [3]. In response to inflammatory stimuli, several response pathways can be initiated, including signal transducers and activators of transcription (STAT) [4], activator protein 1 (AP-1) [5], the nuclear factor of the $\kappa$ light chain enhancer of B cells (NF-kB) [6], and interferon regulatory factors (IRFs) [7]. All these triggers have distinct binding specificities and have different receptor - ligand interaction that leads to a specific transcriptional output. For example, cytokines acting primarily through the activation of STAT TFs such as IFN- $\gamma$, are in general unable to activate NF-KB and AP1 , which are broadly responsive to a large panel of inflammatory stimuli (stretching from LPS and other microbial products to tumor necrosis factor [TNF]- $\alpha$ ] [8]. Inflammation in general, is therefore essential for survival in the adult. Specifically, the blood system responds with cell proliferation, HSC self-renewal and progenitor expansion and differentiation.

Hence, it is all the more striking that these pathways are essential for the HSC generation in the embryo, although the mammalian fetus is protected from pathogens by a robust innate immune system at the maternal/fetal interface [9]. During embryonic development, the first Transplantable HSCs are detected between E10.25-E11.5 in the aorta- gonad and mesophephros region (AGM) [10-12] as residents of Intra-aortic hematopoietic clusters (IAHC) that are closely associated with the ventral wall of the dorsal aorta [13-15]. Although to total number of hematopoietic (cKIT) positive cells in the AGM is believed to total around 600 cells, only a small fraction is HSCs $[10,16]$. In general, blood cells trans-differentiation of specialized endothelial-like cells that can generate blood, termed hemogenic endothelium (HE), to hematopoietic fate during early development. This process is called endothelial to hematopoietic transition (EHT) [17-24]. All the more, it's remarkable that IL-1RI, that is a receptor for IL1 and leads to NF-KB and c-Jun N-terminal kinase (JNK) activation [25], is expressed by cells in the E11 aortic endothelial and mesenchymal cells, and at low levels in the IAHC [26].

Upon stimulation with IL1 $\beta$, AGM explants show higher levels of CXCR4, a chemokine receptor that is specific for stromal cellderived factor-1(SDF-1) required for homing of hematopoietic cell to their niches. The importance of signaling through IL1R1 was further confirmed in transplantation settings; IL1R1 mutant AGM cells showed reduced HSC activity [26]. In fact, recent reports show a correlation of CXCR4 expression and HSC activity in the AGM [27]. In agreement with these findings, Tnf $\alpha$ and TnfR2 morphant zebrafish embryos have decreased expression of the hematopoietic markers runx1 and cmyb, and a NF KB reporter line confirms its activity within the ventral domain of the aorta [28]. Interestingly, the authors find aberrant notch signaling, eg decreased levels of Jag1a, which is required for IAHC formation [28,29]. In parallel, a study on Tlr4, MyD88 and NF KB, a core of inflammatory signaling axis leading to nuclear p65, came to comparable conclusions; they found decreased numbers of hematopoietic cells in the aorta of these morphants, and established a link to aberrant notch signaling in this study, the authors found a reduced induction of the notch 
downstream effectors Hey1/2 and her15.1 [30]. If this lower notch status is due to reduced Jag1a expression as suggested by Espin-Palazon et al. is not known. Interestingly, in the same study, the authors also investigated the HSC activity in the AGM of TLR4 deficient mouse embryos. TLR4 deficient hematopoietic cells in the AGM could generate CFU-C, albeit at a reduced number, but strikingly, almost all HSC activity was diminished in transplantation assays [30]. These studies highlight the high susceptibility of HSC to NF KB signaling, but the HSPCs, the more differentiated cells show a less substantial dependency. Nevertheless, it's still unclear which target genes are activated by p65 mediated NFkB signaling in HSCs and HSPCs.

In an elegant study by Li et al. the authors subjected E10.5 embryos that express GFP under the Ly6a/Sca1 promoter, a marker of HSPCs, including HSCs, to different cytokines [31]. Here they found moderate increases in Sca1 expression after treatment with IL1 $\beta$, IL6 and TnfA in the AGM region, but treatment the Inf $\alpha 4$ or Inf $\gamma$ lead to a huge increase of Sca1 expression in the AGM, including the aorta. The increase in Ly6aGFP+ was due to both, higher expression of Sca1GFP, and enhanced proliferation [31]. In order to determine if there were HSPCs that can receive both Ifn $y$ and Tnfo simultaneously, combinatorial MO knockdown of Ifn $\alpha$ and Tnfy was used in zebrafish embryos. Knockdown of both cytokines together caused a greater decrease in hematopoietic gene expression (runx1) in the dorsal aorta than the knockdown of either gene alone, indicating that multiple inflammatory cytokines can cooperate in the formation and expansion of embryonic HSPCs in the absence of pathological stimulation.

Interestingly, blood flow is needed for the expression of ifng1-2, as ifng1-2 was nearly absent from the axial vessels of tnnt2 (silent heart) morphants that don't have a blood circulation [32]. The reason for this curious observation was not further clarified, but recent studies might provide a plausible explanation. Blood flow might be needed to enhance the sources of inflammatory cytokines to the dorsal aorta.

Detailed analysis of the cellular composition of the AGM microenvironment using mass cytometry (CyTOF) revealed the presence of yolk sac derived macrophages closely associated with IAHC [33]. Prior to HSPC generations in the AGM, the yolk sack produces HSC independent blood progenitors, including macrophages that express different receptors to interact with their niche cells, including Cx3cr1 and Csfr1 [34,35]. Time course analysis with a Csf1r:GFP transgenic mouse model that labels Yolk sac derived macrophages, the study elegantly demonstrates their arrival in the AGM from E9.5 and significantly increasing in abundance by E11.5, ie in time for the HSPC and HSC emergence [33]. Cx3cr1, a chemokine receptor was identified on the macrophages, whereas the possible interacting ligand, Cxcl12, was highly expressed by HE cells. Ablation of $\mathrm{Cx} 3 \mathrm{cr}$, or pharmacological of macrophages with BLZ945, an inhibitor of the colony stimulating factor 1 receptor (CSF1R) reduced CFU-C numbers and HSC activity in transplantation assays. The authors further identified a subpopulation of macrophages based on their cell surface expression of CD206 that specially upregulate cytokine expression of Tnf, Ccl24, Ccl9, Igf1, Bmp2, Pf4, Ccl2, and Ccl7, Whereas Ifno, Ifny, and IL1 $\beta$ expression was observed in all AGM macrophages [33].

In summary, inflammatory signaling pathways are essential for hematopoiesis in the AGM in general, and most importantly, positively influence HSC activity. There is some evidence that some cells are susceptible to more than on the cytokine stimulus at a time [32]. In summary, during development, inflammatory signaling pathways are activated to establish cell identity. It will be important to study the impact of cytokines individually or combined in different cell populations in order to understand the gene expression changes that they inflict. Perhaps the multitude of single cell data sets on AGM cells can give us more clues. Here, we can readily distinguish between the blood cell types and profile their gene expression for inflammatory signaling members. In fact, several single cell data sets of AGM derived cells showed enrichment of inflammatory signaling pathway activation in HE and IAHC [3638]. Further, detailed analysis of these data sets can help to improve our understanding of these stimuli in HSC emergence and helps us to improve in vitro approaches to generate HSC by artificially fine tuning these cytokines in culture conditions.

\section{Conflict of Interest}

No conflict of interest with any institution/organization.

\section{References}

1. Medzhitov R, Horng T (2009) Transcriptional control of the inflammatory response. Nat Rev Immunol 9: 692-703.

2. Natoli G (2010) Maintaining cell identity through global control of genomic organization. Immunity 33: 12-24.

3. Smale ST (2014) Transcriptional regulation in the immune system: a status report. Trends Immunol 35: 190-194.

4. Stark GR, Darnell JE (2012) The JAK-STAT pathway at twenty. Immunity 36: 503-514.

5. Wagner EF, Eferl R (2005) Fos/AP-1 proteins in bone and the immune system. Immunol Rev 208: 126-140.

6. Hayden MS, Ghosh S (2012) NF-kappaB, the first quarter-century: remarkable progress and outstanding questions. Genes Dev 26: 203-234.

7. Tamura T, Yanai H, Savitsky D, Taniguchi T (2008) The IRF family transcription factors in immunity and oncogenesis. Annu Rev Immunol 26: 535-584.

8. O'Shea JJ, Plenge R (2012) JAK and STAT signaling molecules in immunoregulation and immune-mediated disease. Immunity 36: 542-550.

9. Abrahams VM (2008) Pattern recognition at the maternal-fetal interface. Immunol Invest 37: 427-447. 
10. Medvinsky A, Dzierzak E (1996) Definitive hematopoiesis is autonomously initiated by the AGM region. Cell 86: 897-906.

11. Cumano A, Ferraz JC, Klaine M, Di Santo JP, Godin I (2001) Intraembryonic, but not yolk sac hematopoietic precursors, isolated before circulation provide long-term multilineage reconstitution. Immunity 15: 477-485.

12. North TE, De Bruijn MF, Stacy T, Talebian L, Lind E, et al. (2002) Runx1 expression marks long-term repopulating hematopoietic stem cells in the midgestation mouse embryo. Immunity 16: 661-672.

13. De Bruijn MF, Ma X, Robin C, Ottersbach K, Sanchez MJ, et al. (2002) Hematopoietic stem cells localize to the endothelial cell layer in the midgestation mouse aorta. Immunity 16: 673-683.

14. De Bruijn MF, Speck NA, Peeters MC, Dzierzak E (2000) Definitive hematopoietic stem cells first develop within the major arterial regions of the mouse embryo. EMBO J 19: 2465-2474.

15. Jaffredo T, Gautier R, Eichmann A, Dieterlen-Lievre F (1998) Intraaortic hemopoietic cells are derived from endothelial cells during ontogeny. Development 125: 4575-4583.

16. Yokomizo T, Yamada-Inagawa T, Yzaguirre AD, Chen MJ, Speck NA, et al (2012) Whole-mount three-dimensional imaging of internally localized immunostained cells within mouse embryos. Nat Protoc 7: 421-431.

17. Bertrand JY, Chi NC, Santoso B, Teng S, Stainier DY, et al. (2010) Haematopoietic stem cells derive directly from aortic endothelium during development. Nature 464: 108-111.

18. Boisset JC, Van Cappellen W, Andrieu-Soler C, Galjart N, Dzierzak E, et al. (2010) In vivo imaging of haematopoietic cells emerging from the mouse aortic endothelium. Nature 464: 116-120.

19. Eilken HM, Nishikawa S, Schroeder T (2009) Continuous single-cell imaging of blood generation from haemogenic endothelium. Nature 457: 896-900.

20. Kissa K, Herbomel P (2010) Blood stem cells emerge from aortic endothelium by a novel type of cell transition. Nature 464: 112-115.

21. Lam EY, Hall CJ, Crosier PS, Crosier KE, Flores MV (2010) Live imaging of Runx1 expression in the dorsal aorta tracks the emergence of blood progenitors from endothelial cells. Blood 116: 909-914.

22. Lancrin C, Sroczynska P, Stephenson C, Allen T, Kouskoff V, Lacaud G (2009) The haemangioblast generates haematopoietic cells through a haemogenic endothelium stage. Nature 457: 892-895.

23. Percin GI, Eitler J, Kranz A, Fu J, Pollard JW, et al. (2018). CSF1R regulates the dendritic cell pool size in adult mice via embryo-derived tissue-resident macrophages. Nat Commun 9: 5279.

24. Zovein AC, Hofmann JJ, Lynch M, French WJ, Turlo KA, et al. (2008) Fate tracing reveals the endothelial origin of hematopoietic stem cells. Cell Stem Cell 3: 625-636.

\section{ISSN: 2574-1241}

DOI: 10.26717/BJSTR.2021.39.006276

Roshana Thambyrajah. Biomed J Sci \& Tech Res

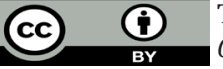

This work is licensed under Creative Commons Attribution 4.0 License

Submission Link: https://biomedres.us/submit-manuscript.php
25. Ishitani T, Takaesu G, Ninomiya-Tsuji J, Shibuya H, Gaynor RB, et al. (2003). Role of the TAB2-related protein TAB3 in IL-1 and TNF signaling. EMBO J 22: 6277-6288.

26. Orelio C, Haak E, Peeters M, Dzierzak E (2008) Interleukin-1-mediated hematopoietic cell regulation in the aorta-gonad-mesonephros region of the mouse embryo. Blood 112: 4895-4904.

27. Dignum T, Varnum-Finney B, Srivatsan SR, Dozono S, Waltner O, et al. (2021) Multipotent progenitors and hematopoietic stem cells arise independently from hemogenic endothelium in the mouse embryo. Cell Rep 36: 109675.

28. Espin-Palazon R, Stachura DL, Campbell CA, Garcia-Moreno D, Del Cid N, et al. (2014) Proinflammatory signaling regulates hematopoietic stem cell emergence. Cell 159: 1070-1085.

29. Robert-Moreno A, Guiu J, Ruiz-Herguido C, Lopez ME, Ingles-Esteve J, et al. (2008) Impaired embryonic haematopoiesis yet normal arterial development in the absence of the Notch ligand Jagged1. EMBO J 27: 18861895.

30. He Q Zhang C, Wang L, Zhang P, Ma D, et al. (2015) Inflammatory signaling regulates hematopoietic stem and progenitor cell emergence in vertebrates. Blood 125: 1098-1106.

31. Ferrajolo C, Coloma PM, Verhamme KM, Schuemie MJ, De Bie S, et al. (2014) Signal detection of potentially drug-induced acute liver injury in children using a multi-country healthcare database network. Drug Saf 37: $99-108$

32. Sawamiphak S, Kontarakis Z, Stainier DY (2014) Interferon gamma signaling positively regulates hematopoietic stem cell emergence. Dev Cell 31: 640-653.

33. Mariani SA, Li Z, Rice S, Krieg C, Fragkogianni S, et al. (2019) Pro-inflammatory Aorta-Associated Macrophages Are Involved in Embryonic Development of Hematopoietic Stem Cells. Immunity 50: 1439-1452 e1435.

34. Canu G, Ruhrberg C (2021) First blood: the endothelial origins of hematopoietic progenitors. Angiogenesis 24: 199-211.

35. Palis J, Yoder MC (2001) Yolk-sac hematopoiesis: the first blood cells of mouse and man. Exp Hematol 29: 927-936.

36. Zeng Y, He J, Bai Z, Li Z, Gong Y, et al. (2019) Tracing the first hematopoietic stem cell generation in human embryo by single-cell RNA sequencing. Cell Res 29: 881-894.

37. Zhou F, Li X, Wang W, Zhu P, Zhou J, et al. (2016) Tracing haematopoietic stem cell formation at single-cell resolution. Nature 533: 487-492.

38. Zhu Q, Gao P, Tober J, Bennett L, Chen C, Uzun Y, et al. (2020) Developmental trajectory of prehematopoietic stem cell formation from endothelium. Blood 136: 845-856

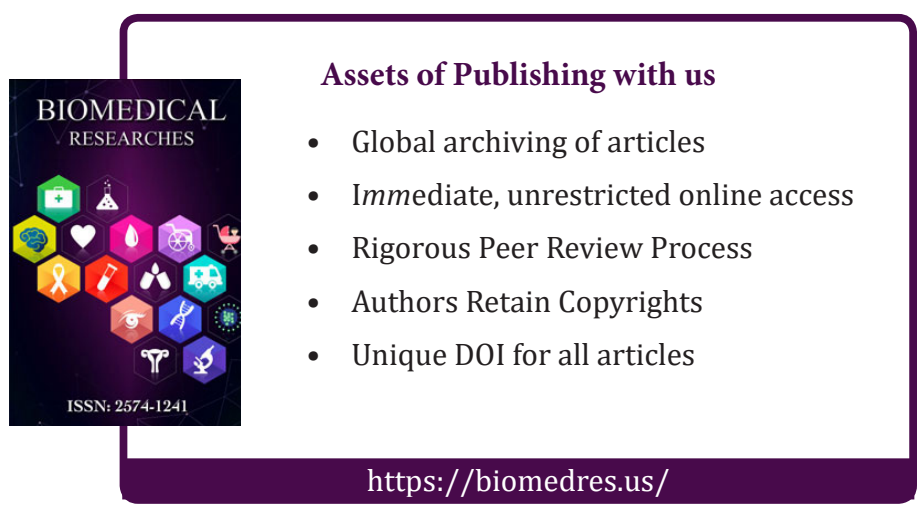

Copyright@ Roshana Thambyrajah | Biomed J Sci \& Tech Res | BJSTR. MS.ID.006276. 